\title{
HELMINTOS PARASITOS DE ATELOPUS BOMOLOCHUS PETERS 1973 (ANURA: BUFONIDAE) DE PIURA, PERU
}

\section{HELMINTHS PARASITES OF ATELOPUS BOMOLOCHUS PETERS 1973 (ANURA: BUFONIDAE) FROM PIURA, PERU}

\author{
José Iannacone \\ Laboratorio de Ecofisiología. Area de Biodiversidad Animal. Facultad de Ciencias Naturales y Matemáticas. \\ Universidad Nacional Federico Villarreal. Calle San Marcos 383, Lima 21, Perú. E-mail: joselorena@terra.com / \\ joseiannacone@hotmail.com
}

\begin{abstract}
RESUMEN
Se realizó una investigación cuantitativa de la fauna parasitaria del anfibio Atelopus bomolochus Peters 1973 (Bufonidae). treintaytres especímenes fueron colectados manualmente de las inmediaciones del río Seco $\left(80^{\circ}\right.$ 9'22" LW, 5 5'19" LS), en las cercanías de la localidad de Chulucanas, provincia de Morropón, departamento de Piura, Perú en octubre del 2000. En total se colectaron 7 especies y 13.268 especímenes. Veintyocho hospederos estuvieron parasitados $(84,8 \%)$. Trece hospederos $(39,4 \%)$ mostraron infección con 1 especie de parásito, seis $(18,2 \%)$, ocho $(24,2 \%)$ y uno $(3,0 \%)$ tuvieron 2,3 y 4 especies de parásitos, respectivamente. Se encontraron siete especies de parásitos con las siguientes prevalencias de infección: Gorgoderina parvicava Travassos 1922 (Digenea) (6,1 \%), Cylindrotaenia americana Jewell 1916 (Cestoda) (9,1\%), Batracholandros spectatus (Freitas \& Ibáñez 1962) Freitas \& Ibáñez 1965 (Nematoda) (33,3 \%), Aplectana hylambatis (Baylis 1927) (6,1 $\%)$; Rhabdias sphaerocephala Goodey 1924 (Nematoda) (69,7\%), Capillaria sp. (Nematoda) (39,4\%) y Centrorhynchus sp. (Acantocephala) (3,0 \%). B. spectatus fue la especie dominante $(97,8 \%)$. Sólo se encontró efecto de la longitud con la prevalencia de infección de $R$. sphaerocephala, una especie central. B. spectatus y Capillaria sp. fueron especies secundarias. G. parvicava, C. americana, A. hylambatis y Centrorhynchus sp. fueron especies satélites. Se discute la relación de la helmintofauna con A. bomolochus.
\end{abstract}

Palabras claves: Acantocephala, Atelopus bomolochus, Anura, Cestoda, Helmintos, Nematoda, Trematoda.

\begin{abstract}
A quantitative research of parasitofauna of thirty-three anuran Atelopus bomolochus Peters 1973 collected from Seco river (80 9'22" LW, 5' 5'19" LS), close to locality of Chulucanas, Province of Morropon, Department of Piura, Peru, in October 2000, was conducted. 13268 specimens of parasites and seven species in total during all the survey were collected. 28 hosts were parasited $(84.8 \%) .13$ hosts $(39.4 \%)$ showed infection with 1 parasite species, six $(18.2 \%)$, eight $(24.2 \%)$ and one $(3,0 \%)$ had two, three and four parasites species, respectively. Seven parasite species with the following prevalence of infections were found: Gorgoderina parvicava Travassos 1922 (Digenea) (6.1\%), Cylindrotaenia americana Jewell 1916 (Cestoda) (9.1\%), Batracholandros spectatus (Freitas \& Ibáñez 1962) Freitas \& Ibáñez 1965 (Nematoda) (33.3\%), Aplectana hylambatis (Baylis 1927) (Nematoda) (6.1 \%), Rhabdias sphaerocephala Goodey 1924 (Nematoda) (69.7\%), Capillaria sp. (Nematoda) (39.4\%), Centrorhynchus sp. (Acantocephala) $(3.0 \%)$. B. spectatus was the dominant species $(97.8 \%)$. The effect of length with prevalence of infection of $R$. sphaerocephala, a core specie was found. B. spectatus and Capillaria sp. are secondary species. $G$. parvicava, C. americana, A. hylambatis and Centrorhynchus sp. are satellite species. The relationship of helminthes parasites with $A$. bomolochus is discussed.
\end{abstract}

Keywords: Acantocephala, Atelopus bomolochus, Anura, Cestoda, Helminths, Nematoda, Trematoda. 


\section{INTRODUCCION}

El Perú, con 315 especies registradas de anfibios, se encuentra en cuarto lugar a nivel mundial en relación a la riqueza de especies, después de tres países neotropicales como Brasil, Colombia y Ecuador (Rodríguez et al. 1993). Atelopus bomolochus Peters 1973 (Bufonidae) es un anfibio que presenta una distribución geográfica conocida que abarca el departamento de Piura. En la zona de Morropón, Piura, Perú, es usado como animal de laboratorio y como mascota (Rodríguez et al. 1993).

Con relación a la fauna parasitaria de $A$. bomolochus no se han registrado parásitos para el Perú (Tantaleán et al. 1992; Sarmiento et al. 1999). Además, la mayoría de los trabajos realizados hasta la fecha en fauna parasitaria de anfibios son sólo de índole cualitativa y de amplitud de ámbito (Tantaleán \& García 1989; Tantaleán et al. 1992; Floríndez \& Morales 1994; Sarmiento et al. 1999) y no se ha efectuado ningún análisis cuantitativo de las comunidades parasitarias en este anuro.

Este trabajo representa un análisis cuantitativo de las comunidades parasitarias de A. bomolochus con el objetivo de evaluar la influencia del tamaño y sexo del hospedero, sobre las especies componentes de su fauna parasitaria.

\section{MATERIALES Y METODOS}

Se colectaron 33 especímenes de este anuro, A. bomolochus en octubre del 2000 en las inmediaciones de campos de cultivo y regadíos del río Seco (80 9'22" LW, 5 5'19" LS), en las cercanías de la localidad de Chulucanas, provincia de Morropón, departamento de Piura, Perú. Desde el punto de vista geográfico, la zona de colecta pertenece a la Región Neotropical, en el Dominio Amazónico y en la Ecorregión de Yunga (Cabrera $\&$ Willink 1980). Los anfibios fueron colectados en horas diurnas utilizando redes de pesca artesanal. Cada espécimen se llevó al laboratorio en forma individual en bolsas plásticas de 10 x $12 \mathrm{~cm}$ tratando que se mantuvieran en óptimo estado de conservación, siendo sacrificados mediante inmersión en agua tibia a $40{ }^{\circ} \mathrm{C}$, posteriormente fueron identificados siguiendo las recomendaciones de Frost (1985) y examinados parasitológicamente en las siguientes $24 \mathrm{~h}$. Los parásitos se colectaron, fijaron, preservaron, colorearon y montaron siguiendo las recomendaciones de Eiras et al. (2000). Los parásitos fueron aislados empleando microscopio de disección y luego lavados en solución salina. Todos los helmintos fueron procesados usando los procedimientos parasitológicos estandarizados para su identificación al nivel de especie. Los tremátodos, céstodos y acantocéfalos fueron fijados y preservados en AFA (alcohol formol ácido acético), luego coloreados con carmín acético de Semichon y finalmente montados en bálsamo de Canadá. Los nemátodos fueron fijados brevemente en alcohol etílico en ebullición al $70 \%$, guardados en alcohol etílico al $70 \%$ con glicerina al $5 \%$ y finalmente clarificados en lactofenol de Amman y examinados tanto en montajes húmedos temporales en gelatina glicerinada y en montajes permanentes en carmín acético de Semichon con bálsamo de Canadá. El abdomen de cada anuro fue abierto por una incisión longitudinal de la cloaca a la cavidad bucal y para la búsqueda de metazoos parásitos se incluyó intestino delgado y grueso, hígado, pulmón, vejiga urinaria y cavidad celómica. Para la determinación taxonómica de los parásitos se usaron los criterios taxonómicos dados por Amin (1985), Anderson (1992) y Khalil et al. (1994). Especímenes representativos de las especies colectadas fueron depositados en la colección helmintológica del Museo de Historia Natural de la Universidad Nacional Mayor de San Marcos (MUSM-UNMSM), Lima, Perú. Gorgoderina parvicava Travassos 1922 MUSM $\mathrm{N}^{\circ} 1764$. Rhabdias sphaerocephala Goodey 1924 MUSM N¹765. Batracholandros spectatus (Freitas \& Ibáñez 1962) Freitas \& Ibáñez 1965 MUSM $\mathrm{N}^{\circ} 1766$. Aplectana hylambatis (Baylis 1927) MUSM N¹767. Capillaria sp. MUSM N 1768.

Se determinó en los hospederos la longitud del hocico a la cloaca (en $\mathrm{cm}$ ) previo a la necropsia. La longitud de los hospederos se dividió en cinco rangos de aproximadamente $1 \mathrm{~cm}$ cada uno. Estos rangos fueron: 7,6 - 8,5 $(\mathrm{n}=1) ; 8,6-$ $9,5(\mathrm{n}=8) ; 9,6-10,5(\mathrm{n}=20) ; 10,6-11,5(\mathrm{n}=3)$; $11,6-12,5(\mathrm{n}=1)$. Además se determinó el peso (en g) para cada anuro. El sexo fue determinado después de la necropsia, tomando en cuenta los caracteres nupciales tanto externos como internos, examinando la cavidad corporal y específicamente las gónadas.

Para todos los parásitos encontrados se 
determinó la prevalencia, intensidad media y abundancia media por anfibio. El coeficiente de dispersión (CD) empleado se determinó de la relación entre Varianza $\left(\mathrm{S}^{2}\right) /$ Intensidad media. Los aspectos ecológicos de los metazoos parásitos a nivel de sus comunidades parasitarias se hicieron para las especies con prevalencias mayores al $10 \%$, de acuerdo a Esch et al. (1990).

La prueba de t de Student, previa evaluación de homogeneidad de varianzas empleando la prueba de Levene, fue usada para determinar si la longitud de los anuros machos y hembras presentaban diferencias significativas. La influencia de la talla del hospedero en la prevalencia de los parásitos se determinó usando el coeficiente de correlación de Spearman $\left(\mathrm{r}_{\mathrm{s}}\right)$. Este se utilizó nuevamente para determinar la relación del tamaño del hospedero con riqueza específica y para cada parásito. Este coeficiente permitió determinar la relación lineal existente entre la riqueza parasitaria específica y la abundancia total. Se aplicó la prueba $\chi^{2}$ para tablas de contingencia para determinar el grado de dependencia entre el sexo del hospedero y la prevalencia parasitaria. El efecto del sexo en la abundancia media parasitaria se calculó utilizando la prueba de t de Student (Zar 1996).

La terminología ecológica para la prevalencia, abundancia e intensidad media siguió los criterios de Bush et al. (1997). Se usó la terminología de especie central, secundaria y satélite propuesta por Bush \& Holmes (1986). Para el cálculo de las pruebas estadísticas descriptivas e inferenciales se usó el paquete estadístico SPSS 7,5 para Windows 95.

\section{RESULTADOS}

La Tabla I muestra la prevalencia, intensidad y abundancia media de infección de los 7 parásitos encontrados en los 33 hospederos muestreados de $A$. bomolochus. Los tres parásitos con mayor frecuencia de dominancia fueron $B$. spectatus $(97,8 \%)$, luego $R$. sphaerocephala $(1,6$ $\%)$ y Capillaria sp. $(0,4 \%)$. Además en cinco de los parásitos colectados se observa una distribución sobredispersa o contagiosa, pues el coeficiente de dispersión fue mayor a 1 (Tabla II).

TABLA I. Prevalencia, intensidad media, abundancia media de infección y número de los helmintos parásitos encontrados en 33 Atelopus bomolochus de Piura, Perú.

TABLE I. Prevalence, intensity and abundance mean of infection and number of helminths parasites obtained in 33 Atelopus bomolochus from Piura, Peru.

\begin{tabular}{|c|c|c|c|c|c|}
\hline ParAsito & $\begin{array}{c}\text { Prevalencia } \\
\% \\
\text { (Hospederos } \\
\text { infectados) }\end{array}$ & $\begin{array}{c}\text { Intensidad } \\
\text { media } \\
\text { (Rango) }\end{array}$ & $\begin{array}{c}\text { Abundancia } \\
\text { media }\end{array}$ & $\begin{array}{l}\text { Namero de } \\
\text { helmintos } \\
\text { encontrados }\end{array}$ & $\begin{array}{l}\text { Porcentaje de la } \\
\text { comunidad }\end{array}$ \\
\hline \multicolumn{6}{|l|}{ Digenea } \\
\hline Gorgoderina parvicava & $\begin{array}{l}6,1 \\
(2)\end{array}$ & $\begin{array}{c}6 \pm 5,7 \\
(2-10)\end{array}$ & $0,4 \pm 1,8$ & 12 & $<0,1$ \\
\hline \multicolumn{6}{|l|}{ Cestoda } \\
\hline Cylindrotaenia americana & $\begin{array}{l}9,1 \\
(3)\end{array}$ & $\begin{array}{c}2 \pm 1,4 \\
(1-3)\end{array}$ & $0,2 \pm 0,5$ & 4 & $<0,1$ \\
\hline \multicolumn{6}{|l|}{ Nematoda } \\
\hline Aplectana hylambatis & $\begin{array}{l}6,1 \\
(2)\end{array}$ & $\begin{array}{c}5,5 \pm 3,5 \\
(3-8)\end{array}$ & $0,3 \pm 1,5$ & 11 & 0,1 \\
\hline Batracholandros spectatus & $\begin{array}{l}33,3 \\
(11)\end{array}$ & $\begin{array}{l}1.180 \pm 471 \\
(770-2.470)\end{array}$ & $393 \pm 623$ & 12.980 & 97,8 \\
\hline Capillaria sp. & $\begin{array}{l}39,4 \\
(13)\end{array}$ & $\begin{array}{c}3,7 \pm 2,9 \\
(1-12)\end{array}$ & $1,5 \pm 2,6$ & 49 & 0,4 \\
\hline Rhabdias sphaerocephala & $\begin{array}{l}69,7 \\
(23)\end{array}$ & $\begin{array}{c}9,2 \pm 8,9 \\
(1-30)\end{array}$ & $6,4 \pm 8,5$ & 211 & 1,6 \\
\hline \multicolumn{6}{|l|}{ Acantocephala } \\
\hline Centrorhynchus sp. & $\begin{array}{l}3,0 \\
(1)\end{array}$ & $\begin{array}{c}1 \pm 0 \\
(1)\end{array}$ & $0,03 \pm 0,2$ & 1 & $<0,1$ \\
\hline
\end{tabular}


Gayana 67(1), 2003

Tabla II. Coeficiente de dispersión, tipo de especie y localización de los helmintos parásitos en 33 Atelopus bomolochus de Piura, Perú.

Table II. Dispersion index, type of specie and location of helminths parasites from 33 Atelopus bomolochus from Piura, Peru.

\begin{tabular}{|c|c|c|c|}
\hline ParAsito & $\begin{array}{l}\text { Coeficiente } \\
\text { de dispersi } \mathrm{n}\end{array}$ & $\begin{array}{c}\text { Tipo de } \\
\text { Especie* }\end{array}$ & Localizaci $n$ \\
\hline \multicolumn{4}{|l|}{ Digenea } \\
\hline Gorgoderina parvicava & 5,3 & SatØite & $\begin{array}{l}\text { Vejiga } \\
\text { urinaria }\end{array}$ \\
\hline $\begin{array}{l}\text { Cestoda } \\
\text { Cylindrotaenia americana }\end{array}$ & 0,9 & SatØite & $\begin{array}{l}\text { Intestino } \\
\text { delgado }\end{array}$ \\
\hline \multicolumn{4}{|l|}{ Nematoda } \\
\hline Aplectana hylambatis & 2,3 & SatØite & $\begin{array}{l}\text { Intestino } \\
\text { grueso }\end{array}$ \\
\hline Batracholandros spectatus & 188 & Secundaria & $\begin{array}{l}\text { Intestino } \\
\text { delgado y } \\
\text { grueso }\end{array}$ \\
\hline Capillaria sp. & 2,2 & Secundaria & $\begin{array}{l}\text { Cavidad } \\
\text { cel mica }\end{array}$ \\
\hline Rhabdias sphaerocephala & 8,6 & Central & Pulmones \\
\hline \multicolumn{4}{|l|}{ Acantocephala } \\
\hline Centrorhynchus sp. & 0 & SatØite & $\mathrm{H}$ gado \\
\hline
\end{tabular}

De los 33 anuros colectados 22 correspondieron a machos y 11 a hembras. Los machos mostraron una longitud de $5,2 \pm 0,6(4,0-6,4)$ y las hembras de 5,5 $\pm 1,0(3,9-7,6)$. El promedio de la longitud de los anuros machos y de las hembras asumiendo igualdad de varianza no fue significa-tivamente diferente $(\mathrm{t}=1,8 ; \mathrm{P}=0,1)$. Además, los machos mostraron un peso de $12,4 \pm 2,1(8,5-18,1)$ y las hembras de $12,3 \pm 2,4(9,8-19,5)$. El promedio del peso de los anuros machos y de las hembras asumiendo igualdad de varianza fue significativamente diferente, siendo las hembras más pesadas que los machos $(\mathrm{t}=$ $3,8 ; \mathrm{P}=0,001)$.

Se observó que sólo la prevalencia de $R$. sphaerocephala mostró relación lineal con la talla del hospedero ( $\left.r_{s}=-0,97, P=0,005\right)$. En cambio, la intensidad y abundancia media de infección no mostraron relación lineal (intensidad media: $\mathrm{r}_{\mathrm{s}}=$ $0,2, \mathrm{P}=0,4$; abundancia media de infestación: $\mathrm{r}_{\mathrm{s}}=$ $0,2, \mathrm{P}=0,2)$. Tampoco se encontró efecto entre el sexo y la prevalencia, intensidad y abundancia media de $R$. sphaerocephala (prevalencia: $\chi^{2}=0,5$,
$\mathrm{P}=0,5$; intensidad media: $\mathrm{t}=0,6, \mathrm{P}=0,6$; abundancia media: $\mathrm{t}=1,1, \mathrm{P}=0,3$ ).

Se notó ausencia de relación lineal entre la talla del hospedero y la prevalencia, intensidad y abundancia media de infección de Capillaria sp. (prevalencia: $\mathrm{r}_{\mathrm{s}}=-0,6, \mathrm{P}=0,3$; intensidad media: $\mathrm{r}_{\mathrm{s}}$ $=-0,02, \mathrm{P}=0,9$; abundancia media: $\mathrm{r}=0,03, \mathrm{P}=0,8$ ). Tampoco se encontró efecto entre el sexo y la prevalencia, intensidad y abundancia media de Capillaria sp. (prevalencia: $\chi^{2}=1,9, \mathrm{P}=0,2$; intensidad media: $\mathrm{t}=0,4, \mathrm{P}=0,7$; abundancia media: $\mathrm{t}=1,5, \mathrm{P}=0,1)$.

Se observó ausencia de relación lineal entre la talla del hospedero y la prevalencia, intensidad y abundancia media de infección de B. spectatus (prevalencia: $\mathrm{r}_{\mathrm{s}}=-0,1, \mathrm{P}=0,9$; intensidad media de infección: $\mathrm{r}_{\mathrm{s}}=-0,3, \mathrm{P}=0,3$; abundancia media de infestación: $\left.\mathrm{r}_{\mathrm{s}}=0,1, \mathrm{P}=0,4\right)$. Tampoco se encontró efecto entre el sexo y la prevalencia, intensidad y abundancia media de $B$. spectatus (prevalencia: $\chi^{2}$ $=0,01, \mathrm{P}=0,9$; intensidad media: $\mathrm{t}=1,9, \mathrm{P}=0,1$; abundancia media: $\mathrm{t}=1,0, \mathrm{P}=0,3$ ). 
Veintiocho $(84,8 \%)$ ranas estuvieron parasitadas por lo menos con una especie de parásito. Un total de 13.268 parásitos fueron colectados, con una abundancia media total de $402 \pm 629(1-2.498)$. La riqueza promedio de las especies de parásitos 1,6 $\pm 1,1(1-4)$ no estuvo relacionada linealmente con la longitud corporal $\left(\mathrm{r}_{\mathrm{s}}=0,3 ; \mathrm{P}=0,1\right)$. Tampoco la abundancia media total estuvo relacionada linealmente con la longitud corporal $\left(\mathrm{r}_{\mathrm{s}}=0,2 ; \mathrm{P}=\right.$ 0,2 ). La abundancia media total y la riqueza de especies estuvieron relacionadas linealmente $(\mathrm{r}=$ $0,9 ; \mathrm{P}=0,0)$. Las infecciones monoespecíficas se encontraron en 13 hospederos $(39,4 \%)$, el biparasitismo en 6 hospederos $(18,2 \%)$, el triparasitismo en 8 hospederos $(24,2 \%)$ y el tetraparasitismo en un hospedero $(3,0 \%)$.

\section{DISCUSION}

En el presente estudio se aislaron siete especies de parásitos: Gorgoderina parvicava Travassos 1922, Cylindrotaenia americana Jewell 1916, Aplectana hylambatis (Baylis 1927), Batracholandros spectatus (Freitas \& Ibáñez 1962) Freitas \& Ibáñez 1965, Capillaria sp., Rhabdias sphaerocephala Goodey 1924 y Centrorhynchus sp.

Barton \& Richard (1996) y Barton (1999) señalan que la composición de la fauna parasitaria de anfibios a nivel mundial muestra bajos niveles de infección y pocas especies, indicando que es una fauna depauperada, en comparación con aves y mamíferos. Barton (1999) señala cuatro factores que pudieran influir en la baja riqueza parasitaria: una dieta oportunista generalizada, un sistema digestivo simple, una baja vagilidad y un metabolismo ectotérmico. Sin embargo, aunque A. bomolochus alberga una riqueza de helmintos menor que en peces, aves y mamíferos, presentó una riqueza específica parasitaria máxima de 7 y promedio de 1,6 , semejante a lo encontrado en muchos anfibios australianos (Barton 1999).

Se detectó que la estructura parasitaria metazoaria de A. bomolochus presentó mayormente carencia de correlación entre la prevalencia, intensidad y abundancia media con el tamaño y sexo de este anuro peruano, en comparación con otros anfibios (Baker 1984; Goldberg et al. 1998; Gillilland \& Muzzall 1999; Muzzall et al. 2001).

En Perú, R. sphaerocephala ha sido regis- trada en cinco especies del género Bufo: Bufo arequipensis Vellard 1959, B. flavolineatus Vellard 1959, B. limensis Werner 1901, B. marinus (Linnaeus 1758) B. trifolium Tschudi 1845 (Sarmiento et al. 1999). B. spectatus ha sido registrada sólo en $B$. limensis y en B. trifolium (Sarmiento et al. 1999). $G$. parvicava ha sido registradas en seis especies y en dos familias de anfibios: Batrachophrynus macrostomus Peters 1863, Bufo limensis, Bufo spinolosus Wiegmann 1835, Leptodactylus rhodonotus (Günther 1868), Telmatobius culeus (Garman 1875) y T. peruvianus Wiegmann 1835 (Tantaleán et al. 1992; Tantaleán \& García 1993; Floríndez \& Morales 1994; Ibáñez 1998). A. hylambatis ha sido registrada en diez especies y en tres familias de anfibios en Perú: Bufo arequipensis, B. flavolineatus, B. marinus, B. spinolosus, B. trifolium, Gastrotheca marsupiata (Duméril \& Bibron 1841), Leptodactylus rhodonotus (Günther 1868), Pleurodema marmorata (Duméril \& Bibron 1841), T. jelskii y T. marmoratus (Duméril \& Bibron 1841) (Floríndez \& Morales 1994, Sarmiento et al. 1999). C. americana, cestodo de amplia distribución en el neotrópico (Rego 1995), ha sido registrada en dos especies y en dos familias de anfibios en Perú: B. spinolosus y T. marmoratus (Tantaleän \& García 1989, Recharte 1995).

Barton (1999) señala que los anuros terrestres están mayormente infectados por un mayor número de nemátodos, en contraste con los anuros acuáticos que albergan mayor cantidad de digéneos, que tienen estadios larvales acuáticos. Esto explica por qué el bufonido A. bomolochus, que es una especie de anfibio de comportamiento mayormente terrestre, alberga cuatro especies de nematodos de un total de siete especies encontradas. En contraste sólo se encontró un digéneo con una prevalencia sumamente baja (Tabla I).

El ciclo biológico $R$. sphaerocephala, la especie más prevalente en $A$. bomolochus, tiene dos generaciones sexuales, una dioica en el suelo de vida libre y otra hermafrodita parásita en los pulmones del anuro. Los adultos machos y hembras copulan en el suelo y la hembra ovovivípara produce larvas filariformes, las cuales infectan al anuro al penetrar por la piel y son transportados por el sistema linfático a todo el cuerpo. Cuando la larva alcanza los pulmones presenta un desarrollo protándrico, luego producen huevos que por vía bronquial alcanzan el sistema digestivo donde eclosionan las larvas 
rabditiformes, las cuales son eliminadas con las heces al suelo y producen la generación dioica (Goldberg et al. 1998).

Los hospederos definitivos de Centrorhynchus son aves rapaces nocturnas (Falconiformes o Strigiformes). Sus hospederos intermediarios son isópodos terrestres, o insectos del grupo de los Orthoptera o Coleoptera, y varios de sus hospederos paraténicos son anfibios, reptiles y mamíferos, que juegan un rol fundamental en la transmisión a aves (Torres \& Puga 1996). A. bomolochus sirve como hospedero paraténico para Centrorhynchus, sin embargo sus hospederos definitivos e intermediarios son desconocidos.

Baker (1984) y Gillilland \& Muzzall (1999) señalan que la abundancia y la riqueza de especies de parásitos están significativamente correlacionadas con la longitud del anfibio hospedero. En A. bomolochus sólo se encontró relación entre su longitud y la prevalencia de $R$. sphaerocephala. Estos resultados sugieren la existencia de ligeras modificaciones en las relaciones ecológicas al incrementar la longitud del hospedador (Marcogliese 2001, Esch \& Fernández 1993, Brooks et al. 2001). Aunque la biología de A. bomolochus sea poco conocida, es probable que este patrón se aplique a este anuro.

Gillilland \& Muzzall (1999) indican que la preferencia a uno de los sexos de los hospederos pudiera ser atribuida a las diferencias en las relaciones ecológicas (comportamiento, hábitat y dieta) de los peces machos y hembras. Nuestros resultados muestran ausencia de efecto del sexo del hospedero sobre la prevalencia y abundancia media parasitaria. En el presente trabajo se repite el mismo patrón encontrado en anuros, donde la mayoría de las especies no mostraron diferencias en la prevalencia y abundancia parasitaria con relación al sexo del hospedero (Yoder \& Coggins 1996, Mc Alpine 1997, Barton 1999, Goldberg et al. 2002).

Este trabajo es el primer reporte de $R$. sphaerocephala, B. spectatus, G. parvicava, A. hylambatis, C. americana, Capillaria sp. y Centrorhynchus sp. en A. bomolochus.

\section{AGRADECIMIENTOS}

El autor expresa su agradecimiento al Laboratorio de Ecofisiología de la Facultad de
Ciencias Naturales y Matemáticas de la Universidad Nacional Federico Villarreal por su apoyo a la presente investigación. A los estudiantes de biología Richard Huaraz, José Soto y Rosario Reyes por su apoyo en la colecta de los anfibios. A Helena Sisniegas (Consejo Nacional de Ciencia y Tecnología, Lima, Perú) por la identificación de la especie de anfibio. Este trabajo fue expuesto en el V Congreso Peruano de Parasitología, Trujillo, Perú del 2 al 5 de octubre del 2002. Al Consejo Nacional de Ciencia y Tecnología (CONCYTEC), Lima, Perú, por el auspicio de la presente investigación.

\section{BIBLIOGRAFIA}

ANDERSON. R.C. 1992. Nematode parasites of vertebrates: their development and transmission. CAB International, Cambridge.

Amin, O. 1985. Biology of Acantocephala. pp. 27-72. In: D.W.T. Crompton; BB. Nickol. Cambridge.

BAKER, M. R. 1984. Nematode parasitism in amphibian and reptiles. Can. J. Zool. 62: 747-757.

Barton, D. P. \& S. J. Richards. 1996. Helminth infracommunities in Litoria geniculata (Amphibia: Anura) from Birthday Creek, an upland rainforest stream in northern Queensland, Australia. Int. J. Parasitol. 26: 1381-1385.

BARTON, D. P. 1999. Ecology of helminth communities in tropical Australian amphibians. Int. J. Parasitol. 29: 921-926.

Brooks, D. R., V. León-Regagnon \& G. Pérez-Ponce. 2001. Los parásitos y la biodiversidad. pp. 245289. In H. M. Hernández, A.N. García, F. Alvarez \& M. Ulloa (Comps.). Enfoques contemporáneos para el estudio de la biodiversidad. Instituto de Biología, UNAM. México.

Bush, A.O. \& J.C. Holmes. 1986. Intestinal helminthes of lesser scaup ducks: an interactive community. Can. J. Zool. 64: 142-152.

Bush, A.O., K.D. Lafferty, J.L. Lotz \& A.W. Shostak. 1997. Parasitology meets ecology on its own terms: Margolis et al. revisited. Jour. Parasitol. 83: 575-583.

Cabrera, D. \& R. Willink. 1980. Biogeografía de América Latina. Monografía No 13. OEA. 122 p.

Eiras, J., R. TAKemoto \& G. C. Pavanelli. 2000. Métodos de estudo e técnicas laboratoriais em parasitología de peixes. Universidade Estadual de Maringá (Ed). Maringa. 171 pp.

Esch, G. W. \& J. C. FERnÁndEz. 1993. A functional biology of parasitism: Ecological and evolutionary implications. Chapman \& Hall. Cambridge.

Esch, G.W., A.W. ShostaK, D.J. \& T.M. Goater. 1990. Patterns and process in helminth parasite communities: an overview. pp. 1-19. In G. Esch, A.C. Bush \& J. Aho. (Eds). Parasite Communities: Pat- 
terns and processes. Chapman and Hall. New York. Floríndez, D. \& M. E. Morales. 1994. Helmintofauna de Leptodactylus rhodonotus (Anura: Leptodactylidae) de Oxapampa, Pasco, Perú. Libro de Resúmenes de la IV Reunión Científica del Instituto de Investigación de Ciencias Biológicas “Antonio Raimondi”(ICBAR), Universidad Nacional Mayor de San Marcos. p. 61.

Frost, D. 1985. Amphibian species of the World. Allen Press, Lawrence, Kansas. 732 p.

Gillilland, M.G. \& P.M. Muzzall. 1999. Helminths infecting froglets of the northern leopard frog (Rana pipiens) from Foggy Botton Marsh, Michigan. Jour. Helminth. Soc. Wash. 66: 7377.

Goldberg, S. R., C.R. Bursey \& H. Kaiser. 1998. Gastrointestinal helminthes of the species of Eleutherodactylus (Anura: Leptodactylidae) from West Indies. Carib. J. Sci. 34: 146-149.

Goldberg, S.R., C.R. Bursey, J.D. Trujillo \& H. KaISER. 2002. Intestinal helminths of seven frog species from Trinidad and Tobago. Carib. J. Sci. 38: 147-150.

IBÁÑEZ, N. H. 1998. Mención de algunos trematodos en la fauna helmintológica peruana. Rev. Perú. Parasitol. 13: 90-97.

KhaliL, L.F.; A. Jones \& R.A. Bray. 1994. Keys to the cestode parasites of vertebrates. CAB International, Wallingford. $751 \mathrm{p}$.

Marcogliese, D. J. 2001. Pursuing parasites up the food chain: implications of food web structure and function on parasite communities in aquatic systems. Acta Parasitol. 46: 82-93.

Mc Alpine, D.F. 1997. Helminth communities in bullfrogs (Rana catesbeiana), green frogs (Rana clamitans), and leopard frogs (Rana pipiens) from New Brunswick, Canada. Can. J. Zool. 75: 1883-1890.

Muzzall, P.M., M. G. Gillilland, C.S. Summer \& C.J. MehNe. 2001. Helminths communities of green frogs Rana clamitans Latreille, from southwestern Michigan. Jour. Parasitol. 87: 962-968.

Recharte, A. 1995. Parásitos de Bufo spinolosus Weigmann, 1835 y Telmatobius marmoratus pseudojleskii Wiegmann, 1834 de la granja K'ayra, Cusco. Biotempo (Perú) 2: 87-90.

Rego, A.A. 1995. A new classification of the cestode Order Proteocephalidea Mola. Revta. Bras. Zool. 12: 791-814.

Rodríguez, L.O., J. H. Córdova \& J. ICOCHEA. 1993. Lista preliminar de los anfibios del Perú. Publ. Mus. Hist. Nat. UNMSM (A) 45: 1-22.

Sarmiento, L., M. Tantaleán \& A. Huiza. 1999. Nemátodos parásitos del hombre y de los animales en el Perú. Rev. Peru. Parasitol. 14: 965.

Tantaleán, M. V. \& L. García. 1989. Contribución al estudio de los helmintos parásitos de anfibios del Perú. Bol. Lima (Perú) 64: 69-78.

Tantaleán, M.V., L. Sarmiento \& A. Huiza. 1992. Digeneos (Trematoda) del Perú. Bol. Lima (Perú) 80: 47-84.

Tantaleán, M.V. \& L. García. 1993. Trematodes de la familia Gorgoderidae en anfibios Leptodactylidae de la región altoandina del Perú. Bol. Lima (Perú) 85: 25-27.

Torres, P. \& S. Puga. 1996. Occurence of cystacanths of Centrorhynchus sp. (Acantocephala: Centrorhynchidae) in toads of the genus Eupsophus in Chile. Mem. Inst. Oswaldo Cruz 91: 717-719.

Yoder, H.R. \& J.R. Coggins. 1996. Helminth communities in the northern spring peeper, Pseudacris c. crucier Wied, and the wood frog, Rana sylvatic Le Conte from Southeastern Wisconsin. Jour. Helminth. Soc. Wash.63: 211-214.

ZAR, J.H. 1996. Biostatistical Analysis. $3^{\text {th }}$ Ed. Prentice-Hall, Inc. Upper Saddle River. New Jersey. 662 pp. 